\title{
R-matrix calculation of the continuum states of Carbon Monoxide
}

\author{
K. Chakrabarti ${ }^{\dagger}$ and Jonathan Tennyson* \\ †Department of Mathematics, Scottish Church College, 1 \& 3 Urquhart Sq., Kolkata \\ 700006, India \\ *Department of Physics and Astronomy, University College London, Gower St., \\ London WC1E 6BT, UK
}

\begin{abstract}
Calculations on electron collisions with $\mathrm{CO}^{+}$molecular ion are presented as a function of electron energy and $\mathrm{CO}^{+}$geometry. Resonance positions and widths are obtained for the low-lying Feshbach resonances in the system. Plots of resonance curves suggest that low-energy dissociative recombination can occur via resonances of ${ }^{1} \Pi,{ }^{1} \Delta,{ }^{3} \Sigma^{+}$and ${ }^{3} \Pi$ symmetries. Electron impact electronic excitation is considered both for excitation to the first excited a ${ }^{2} \Pi$ state and to yield a dissociation cross section. The latter calculations suggest that the lower of two experimental measurements of this process is likely to be correct. Finally results are presented for bound states in the continuum for both ${ }^{1} \Sigma^{-}$and ${ }^{3} \Sigma^{-}$symmetries.
\end{abstract}

\section{Introduction}

Carbon monoxide is the second most abundant molecule in the Universe and its ion, $\mathrm{CO}^{+}$, has been observed in a variety of astronomical locations (Erickson et al. 1981, Storzer et al. 1995, Fuente \& Martin Pintado 1997, Fox \& Hac 1999). Given that such ions occur in plasmas, collisions with low-energy electrons are likely to be important. In particular low-energy electron collisions can lead to both (de-)excitation of the ion and to its destruction.

At low collision energies dissociative recombination (DR) is the major route to destruction for molecular ions. Low-energy $\mathrm{DR}$ rates for $\mathrm{CO}^{+}$have been measured both in merged beams (Mitchell \& Hus 1985) and storage rings (Rosen et al. 1998). Models of dissociative recombination require both accurate resonance curves and associated widths, and a detailed treatment of the nuclear motion problem (Schneider et al. 2000, Florescu-Mitchell \& Mitchel 2006). Here we provide the curves that could provide a starting point for such a treatment.

Besides DR studies there are few studies of electron $-\mathrm{CO}^{+}$collisions. A recent measurement focused on electron impact ionisation (Lecointre et al. 2006), and the two cited DR studies (Mitchell \& Hus 1985, Rosen et al. 1998) also presented results for electron impact dissociation which were not in good agreement with each other. 
Rather surprisingly, given its importance, we know of no previous theoretical study of continuum states of $\mathrm{CO}^{+}$.

In an earlier study (Chakrabarti \& Tennyson 2006), we used the R-matrix method to present a comprehensive study of the Rydberg states of the CO. In this work we consider the low-lying continuum of the CO system placing particular emphasis on those resonances that are likely to be important for dissociative recombination. We also consider processes involving electron impact excitation. The following section outlines the general method and details the calculations. Results are presented in section 3 and the final section presents our conclusions.

\section{Calculations}

\subsection{Method}

The details of the calculations are already presented in our earlier work (Chakrabarti \& Tennyson 2006) so we present here only the essentials. The R-matrix method divides configuration space into two regions (Burke \& Berrington 1993). The inner region is defined by a sphere, here of radius $10 a_{0}$, centred at the molecular centre of mass. This sphere encloses the entire $N$-electron target wave function, where for present purposes the 'target' means $\mathrm{CO}^{+}$. In this inner region, the wave function of the $(N+1)$-electron system, CO, is given by

$$
\Psi_{k}=\mathcal{A} \sum_{i, j} a_{i, j, k} \Phi_{i}(1, \ldots, N) F_{i, j}(N+1)+\sum_{i} b_{i, k} \chi_{i}(1, \ldots, N+1),
$$

where $\mathcal{A}$ is the anti-symmetrisation operator, $F_{i, j}$ are continuum orbitals and $\chi_{i}$ are twocentre $L^{2}$ functions constructed from $N$-electron 'target' orbitals. Also $\Phi_{i}$ is the wave function of the $i^{\text {th }}$ target state. Electron-correlation effects are included in these target wave functions via configuration interaction (CI) expansions (Tennyson 1996a). The variational coefficients $a_{i, j, k}$ and $b_{i, k}$ are determined by diagonalising the Hamiltonian matrix. For diatomic calculations the inner region code is based on the Alchemy (McLean 1971) quantum chemistry package.

\section{2. $\mathrm{CO}^{+}$target}

Calculations on both $\mathrm{CO}^{+}$and $\mathrm{CO}$ were performed for 10 internuclear separations in the range $1.5-3.5 \mathrm{a}_{0}$. The CO Slater-type orbitals of Kirby-Docken \& Liu (1977) were used to build a $\mathrm{CO}^{+}$molecular basis of 46 molecular orbitals $(24 \sigma, 14 \pi, 6 \delta, 2 \phi)$. An initial set of molecular orbitals was obtained by performing self-consistent field (SCF) calculations on the two lowest states of $\mathrm{CO}^{+}, \mathrm{X}^{2} \Sigma^{+}$and $\mathrm{A}^{2} \Pi$. These SCF molecular orbitals were then used in a configuration interaction (CI) calculation. In all calculations the $1 \sigma$ and $2 \sigma$ molecular orbitals were frozen and the CI calculation used the following configurations:

$(3 \sigma, 4 \sigma, 5 \sigma, 1 \pi)^{9}$, $(3 \sigma, 4 \sigma, 5 \sigma, 1 \pi)^{8}(6 \sigma-24 \sigma, 2 \pi-14 \pi, 1 \delta-6 \delta)^{1}$, 
$(3 \sigma, 4 \sigma, 5 \sigma, 1 \pi)^{7},(6 \sigma-24 \sigma, 2 \pi-14 \pi, 1 \delta-6 \delta)^{2}$.

These correspond, respectively, to a complete active space (CAS) spanning valence orbitals, single excitations from this CAS and double excitations. Two sets of pseudonatural orbitals (NOs) were obtained from CI calculation on the lowest $\mathrm{CO}^{+}$states with ${ }^{2} \Sigma^{+}$and ${ }^{2} \Pi$ symmetry described above. Following Tennyson (1996b), all $\sigma$ and the $1 \pi$ orbital in the target state wave function were represented by ${ }^{2} \Sigma^{+}$NOs, and the remaining $\pi$ and all $\delta$ orbitals were represented by the ${ }^{2} \Pi$ NOs.

Our model uses the NOs constructed as above, 4 electrons frozen the $1 \sigma^{2}$ and $2 \sigma^{2}$ orbitals corresponding to the $\mathrm{O}$ and $\mathrm{C} 1$ s electrons respectively, and a $(3 \sigma, 4 \sigma, 5 \sigma, 6 \sigma$, $1 \pi, 2 \pi)^{9}$ CAS-CI (note that in our earlier work the CAS used was wrongly quoted as $(3 \sigma$, $\left.4 \sigma, 5 \sigma, 1 \pi)^{9}\right)$. The $\mathrm{CO}^{+}$excitations were compared by Chakrabarti \& Tennyson (2006) with the experimental results of Huber \& Herzberg (1979) and the CCSDT calculation of Okada \& Iwata (2000) and were generally found to be in good agreement with our work. Calculated potential energy curves for the 14 target states used in the close coupling expansion can also be found in our previous paper.

\subsection{The $C O$ model}

Our calculations used $14 \mathrm{CO}^{+}$natural orbitals $(8 \sigma, 4 \pi, 2 \delta)$. These orbitals were augmented by continuum orbitals $F_{i, j}$, expressed as a truncated partial wave expansion around the centre of mass. Partial waves with $l \leq 6$ and $m \leq 2$ were retained in the expansion. The radial parts of the continuum functions were generated as numerical solutions of an isotropic Coulomb potential. Those solutions with an energy below 10 Ryd were retained. A Buttle (1967) correction was used to correct for the effect of this truncation or, alternatively, the fixed boundary condition used to generate the functions. To correct for linear dependence effects two $\sigma$ and one $\pi$ orbitals were removed using Lagrange orthogonalisation (Tennyson et al. 1987). The resulting $62 \sigma, 52 \pi, 42 \delta$ functions were Schmidt orthogonalised to the target NOs.

Calculations were performed using the $(3 \sigma-6 \sigma, 1 \pi, 2 \pi)^{9}$ CAS target wave function for the $\mathrm{CO}^{+}$states, up to 14 of which were used in the close-coupling expansion (1). Further details of the calculation, including a tabulation of the target states and energies involved, can be found in our previous study (Chakrabarti \& Tennyson 2006). Calculations were performed for the lowest four symmetries of both singlet and triplet symmetry meaning that the resonances we consider are of total symmetry ${ }^{1} \Sigma^{+},{ }^{1} \Pi$, ${ }^{1} \Delta,{ }^{1} \Sigma^{-},{ }^{3} \Sigma^{+},{ }^{3} \Pi,{ }^{3} \Delta$ and ${ }^{3} \Sigma^{-}$. Resonance series belonging to angular momentum symmetries $\Phi$ or higher will be both of higher energy and have narrower resonance meaning that these resonances are of less physical significance. As we only consider electronic excitation here, no attempt was made to top up the calculation for neglected symmetries. Experience shows that these higher symmetries, while important for elastic scattering, do not contribute significantly to electron impact electronic excitation cross sections (Gorfinkiel et al 2002). 


\subsection{Resonances}

The inner region solutions, eq. (1), were used to construct $R$-matrices on the boundary. In the outer region, in addition to the Coulomb potential, the potential was given by the diagonal and off-diagonal dipole and quadrupole moments of the $\mathrm{CO}^{+}$target states. The $R$-matrices were propagated (Morgan 1984) in this potential until the wave function could be matched with exponentially decreasing functions obtained from a Gailitis expansion (Noble \& Nesbet 1984). Tests showed that propagating the $R$-matrices to $100 \mathrm{a}_{0}$ gave stable results and this value was used for all calculations presented here. Not all target states included in the inner region close-coupling expansion were included in the outer region calculation as those which are strongly closed were found to make negligible difference in the calculation.

Resonances were detected and fitted to a Breit-Wigner profile to obtain their energy $\left(E_{r}\right)$ and width $(\Gamma)$ using the RESON program (Tennyson \& Noble 1985) with an energy grid $0.25 \times 10^{-3} \mathrm{Ryd}$. The magnitudes of the complex quantum defects $\mu=\alpha+i \beta$ were obtained using the relations

$$
E_{r}=E_{t}-\frac{1}{\nu^{2}}, \quad \Gamma=\frac{2 \beta}{\nu^{3}}
$$

where the effective quantum number, $\nu$, equals $n-\alpha$ and $E_{t}$ is the energy of the threshold to which the Rydberg series converges.

Although this work is confined to energies higher than the CO ionisation limit some of the states we find are bound within our model. These states are of $\Sigma^{-}$electronic symmetry which cannot couple to the $\mathrm{X}^{2} \Sigma^{+}$ground state of $\mathrm{CO}^{+}$by loss of a single electron. Energies of these states thus appear as bound states associated with the Rydberg series converging on the $\mathrm{A}^{2} \Pi$ first excited state of $\mathrm{CO}^{+}$. These bound states were found using program BOUND which employs the searching algorithm of Sarpal et al. (1991) with an improved nonlinear, quantum defect based grid (Rabadán \& Tennyson 1996).

\section{Results}

\subsection{Resonances}

There is clearly a considerable quantity of data available for the resonances from these calculations so all we can do here is present a summary. Further information or numerical values for data presented in the figures can be obtained from the authors.

Tables 1 and 2 present data on the resonances obtained for the $\mathrm{CO}$ equilibrium bondlength of $2.132 \mathrm{a}_{0}$ for singlet and triplet total symmetries respectively. In each case we tabulate values for resonance series converging on the lowest two electronically excited for which coupling is allowed. For the triplets these correspond to resonances associated with the $\mathrm{A}^{2} \Pi$ and a ${ }^{4} \Sigma^{+}$states respectively, which are indeed the lowest two excited states of $\mathrm{CO}^{+}$in the equilibrium region. However the singlet resonances cannot 
Table 1. Resonance positions and widths (in Ryd), and effective quantum numbers $(\nu)$ at $R=2.132 \mathrm{a}_{0}$ for singlet total symmetries of the $\mathrm{CO}$ molecule below the first two $\mathrm{CO}^{+}$excited states. Numbers within brackets indicate power of 10 .

\begin{tabular}{|c|c|c|c|c|c|}
\hline \multicolumn{3}{|c|}{ Below ${ }^{2} \Pi$ state } & \multicolumn{3}{|c|}{ Below $^{2} \Delta$ state } \\
\hline Position & Width & $\nu$ & Position & Width & $\nu$ \\
\hline \multicolumn{6}{|l|}{${ }^{1} \Sigma^{+}$} \\
\hline 0.1090 & $0.2068(-02)$ & 2.3638 & 0.3172 & $0.3140(-02)$ & 2.4091 \\
\hline 0.1386 & $0.2722(-01)$ & 2.5870 & 0.3712 & $0.1104(-02)$ & 2.9078 \\
\hline 0.1992 & $0.4533(-02)$ & 3.3564 & 0.3852 & $0.4259(-02)$ & 3.0964 \\
\hline 0.2006 & $0.5409(-02)$ & 3.3833 & 0.3873 & $0.4953(-02)$ & 3.1288 \\
\hline 0.2254 & $0.1355(-04)$ & 3.9970 & 0.4059 & $0.9631(-03)$ & 3.4600 \\
\hline 0.2317 & $0.1193(-02)$ & 4.2135 & 0.4237 & $0.4112(-03)$ & 3.8988 \\
\hline 0.2368 & $0.7869(-03)$ & 4.4206 & 0.4269 & $0.4054(-04)$ & 3.9967 \\
\hline 0.2477 & $0.6932(-04)$ & 4.9834 & 0.4308 & $0.1285(-02)$ & 4.1292 \\
\hline 0.2540 & $0.2918(-03)$ & 5.4211 & 0.4393 & $0.4684(-03)$ & 4.4664 \\
\hline 0.2580 & $0.8255(-03)$ & 5.7791 & 0.4477 & $0.1802(-03)$ & 4.8921 \\
\hline 0.2637 & $0.1455(-03)$ & 6.4203 & 0.4515 & $0.6514(-03)$ & 5.1352 \\
\hline 0.2654 & $0.9502(-03)$ & 6.6568 & 0.4560 & $0.2608(-03)$ & 5.4718 \\
\hline 0.2698 & $0.9090(-04)$ & 7.4192 & 0.4606 & $0.9713(-04)$ & 5.8889 \\
\hline \multicolumn{6}{|l|}{${ }^{1} \Pi$} \\
\hline 0.0376 & $0.2137(-03)$ & 1.9983 & 0.3076 & $0.6750(-03)$ & 2.3451 \\
\hline 0.1155 & $0.7100(-03)$ & 2.4076 & 0.3852 & $0.6825(-03)$ & 3.0973 \\
\hline 0.1696 & $0.4020(-03)$ & 2.9068 & 0.4008 & $0.1995(-03)$ & 3.3594 \\
\hline 0.1772 & $0.7000(-03)$ & 3.0044 & 0.4216 & $0.3425(-02)$ & 3.8404 \\
\hline 0.1847 & $0.1181(-03)$ & 3.1113 & 0.4307 & $0.5004(-03)$ & 4.1266 \\
\hline 0.2035 & $0.2352(-04)$ & 3.4398 & 0.4370 & $0.7934(-04)$ & 4.3660 \\
\hline 0.2219 & $0.1150(-03)$ & 3.8897 & 0.4514 & $0.1994(-03)$ & 5.1235 \\
\hline 0.2375 & $0.7976(-05)$ & 4.4508 & 0.4547 & $0.4684(-04)$ & 5.3672 \\
\hline 0.2500 & $0.1713(-04)$ & 5.1335 & 0.4648 & $0.3093(-04)$ & 6.3676 \\
\hline $\begin{array}{c}0.2591 \\
{ }^{1} \Delta\end{array}$ & $0.2736(-04)$ & 5.8801 & & & \\
\hline 0.1110 & $0.5150(-03)$ & 2.3769 & 0.3402 & $0.9520(-03)$ & 2.5886 \\
\hline 0.1969 & $0.3472(-03)$ & 3.3136 & 0.3546 & $0.1335(-01)$ & 2.7227 \\
\hline \multirow[t]{4}{*}{0.2023} & $0.1848(-04)$ & 3.4168 & 0.3782 & $0.8065(-03)$ & 2.9985 \\
\hline & & & 0.4264 & $0.1065(-03)$ & 3.9810 \\
\hline & & & 0.4495 & $0.9671(-05)$ & 5.0005 \\
\hline & & & 0.4689 & $0.9671(-05)$ & 6.9708 \\
\hline
\end{tabular}


Table 2. Resonance positions and widths (in Ryd), and effective quantum numbers $(\nu)$ at $R=2.132 \mathrm{a}_{0}$ for triplet total symmetries of the CO molecule below the first two $\mathrm{CO}^{+}$excited states. Numbers within brackets indicate power of 10 .

\begin{tabular}{|c|c|c|c|c|c|}
\hline \multicolumn{3}{|c|}{ Below ${ }^{2} \Pi$ state } & \multicolumn{3}{|c|}{ Below ${ }^{4} \Sigma^{+}$state } \\
\hline Position & Width & $\nu$ & Position & Width & $\nu$ \\
\hline \multicolumn{6}{|l|}{${ }^{3} \Sigma^{+}$} \\
\hline 0.09116 & $0.3450(-03)$ & 2.2540 & 0.3053 & $0.5747(-02)$ & 2.3304 \\
\hline 0.1790 & $0.1572(-03)$ & 3.0288 & 0.3143 & $0.1194(-02)$ & 2.3895 \\
\hline 0.1950 & $0.1946(-03)$ & 3.2787 & 0.3574 & $0.2796(-02)$ & 2.7520 \\
\hline 0.2216 & $0.7724(-03)$ & 3.8817 & 0.3689 & $0.1177(-02)$ & 2.8800 \\
\hline 0.2297 & $0.2507(-03)$ & 4.1411 & 0.3822 & $0.9634(-03)$ & 3.0541 \\
\hline 0.2347 & $0.1450(-03)$ & 4.3323 & 0.4040 & $0.3654(-03)$ & 3.4207 \\
\hline 0.2525 & $0.3858(-04)$ & 5.3090 & 0.4155 & $0.3577(-03)$ & 3.6773 \\
\hline 0.2628 & $0.2102(-04)$ & 6.3058 & 0.4226 & $0.4015(-03)$ & 3.8664 \\
\hline \multirow[t]{6}{*}{0.2675} & $0.3713(-05)$ & 6.9934 & 0.4268 & $0.4016(-04)$ & 3.9959 \\
\hline & & & 0.4290 & $0.3768(-03)$ & 4.0656 \\
\hline & & & 0.4386 & $0.1709(-03)$ & 4.4329 \\
\hline & & & 0.4471 & $0.2020(-03)$ & 4.8608 \\
\hline & & & 0.4506 & $0.2287(-03)$ & 5.0742 \\
\hline & & & 0.4556 & $0.9297(-04)$ & 5.4380 \\
\hline \multicolumn{6}{|l|}{${ }^{3} \Pi$} \\
\hline 0.0191 & $0.6342(-03)$ & 1.9286 & 0.3025 & $0.1845(-02)$ & 2.3129 \\
\hline 0.0309 & $0.4981(-03)$ & 1.9723 & 0.3495 & $0.1257(-02)$ & 2.6734 \\
\hline 0.1133 & $0.7167(-03)$ & 2.3925 & 0.3838 & $0.6216(-03)$ & 3.0758 \\
\hline 0.1660 & $0.4748(-03)$ & 2.8627 & 0.3994 & $0.5882(-03)$ & 3.3326 \\
\hline 0.1824 & $0.3661(-03)$ & 3.0771 & 0.4127 & $0.2164(-02)$ & 3.6097 \\
\hline 0.1859 & $0.1193(-02)$ & 3.1303 & 0.4269 & $0.6898(-04)$ & 3.9974 \\
\hline 0.1992 & $0.1251(-03)$ & 3.3561 & 0.4297 & $0.1301(-03)$ & 4.0910 \\
\hline 0.2031 & $0.2435(-03)$ & 3.4332 & 0.4364 & $0.2987(-03)$ & 4.3436 \\
\hline 0.2205 & $0.1118(-03)$ & 3.8493 & 0.4510 & $0.9525(-04)$ & 5.0974 \\
\hline 0.2255 & $0.1138(-04)$ & 4.0011 & 0.4544 & $0.1466(-03)$ & 5.3425 \\
\hline 0.2290 & $0.7073(-04)$ & 4.1158 & 0.4577 & $0.2995(-02)$ & 5.6114 \\
\hline $\begin{array}{c}0.2371 \\
{ }^{3} \Delta\end{array}$ & $0.8348(-04)$ & 4.4330 & 0.4626 & $0.4277(-04)$ & 6.1004 \\
\hline \multirow[t]{5}{*}{0.09416} & $0.4029(-03)$ & 2.2714 & 0.3613 & $0.2080(-03)$ & 2.7935 \\
\hline & & & 0.4120 & $0.1415(-03)$ & 3.5942 \\
\hline & & & 0.4258 & $0.2046(-04)$ & 3.9644 \\
\hline & & & 0.4344 & $0.2562(-03)$ & 4.2620 \\
\hline & & & 0.4487 & $0.1175(-04)$ & 4.9561 \\
\hline
\end{tabular}



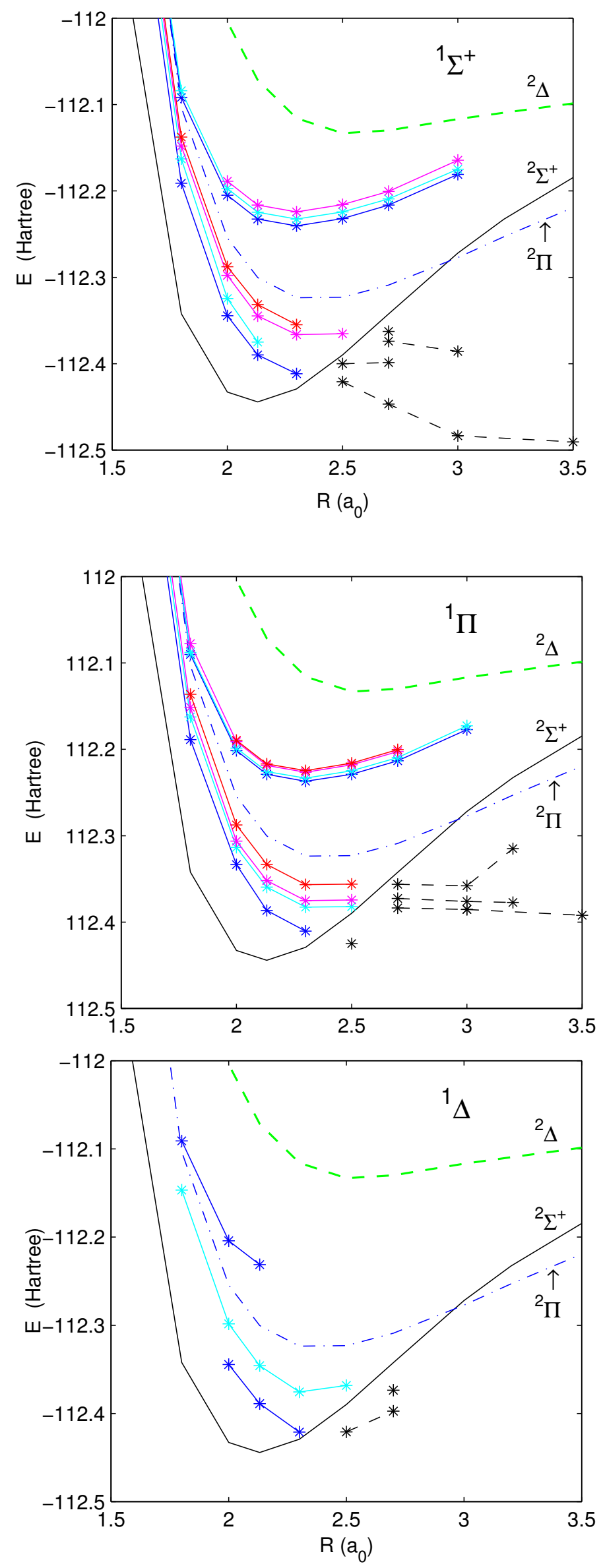

Figure 1. Singlet CO resonance curves as a function of internuclear separation with actual calculated points denoted by stars. The symmetry of each state is indicated in the panel. Also shown are potential energy curves for the three lowest $\mathrm{CO}^{+}$target states of doublet spin symmetry. Resonance which cross the $\mathrm{CO}^{+} \mathrm{X}^{2} \Sigma^{+}$curve are 

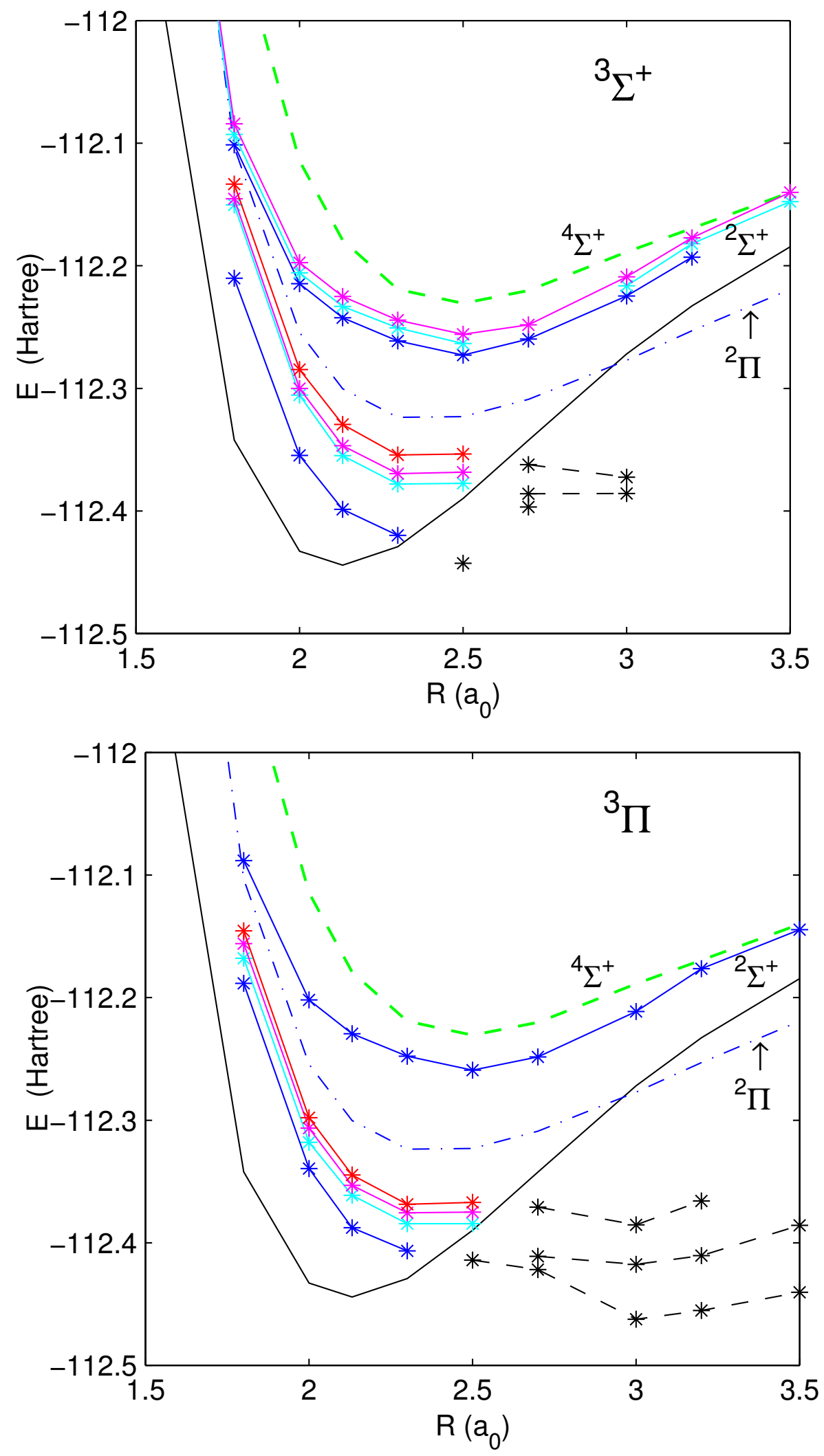

Figure 2. Triplet CO resonance curves as a function of internuclear separation with actual calculated points denoted by stars. The symmetry of each state is indicated in the panel. Also shown are potential energy curves for the three lowest $\mathrm{CO}^{+}$target states. Resonance which cross the $\mathrm{CO}^{+} \mathrm{X}^{2} \Sigma^{+}$curve are bound 
couple to quartet target states so for the singlets the first two resonance series converge to the $\mathrm{A}^{2} \Pi$ and $\mathrm{B}^{2} \Sigma^{+}$states.

Tables 1 and 2 also give the effective quantum number, $\nu$. This parameter is useful for identifying both resonances which form a series and possible intruder states ie resonances which are actually associated with a higher-lying excited state of the ion. Thus for example ${ }^{1} \Sigma^{+}$symmetry resonances show a clear series of resonances with the real part of the quantum defect, $\alpha$, equal to about 0.6. Conversely the resonance at 0.1386 Ryd has a a quantum defect of 0.4 , unlike any other resonance detected in this series, and is almost certainly an intruder or interloper (McLaughlin et al. 2001) state. Such states can also be detected by analysing their behaviour as a function of internuclear separation, $R$. This can be done by either plotting the variation of the quantum defects with $R$ (Rabadán \& Tennyson 1997), or just the resonance curves which is particularly effective if the ionic curves they are associated with behave differently with $R$. Figures 1 and 2 show the behaviour of the low-lying two resonances series as function of $R$ for the symmetries for which we find low-lying resonances.

The most important resonances for dissociative recombination (DR) studies are those low-lying ones which lead to dissociation by crossing the target ion ground state potential energy curve at energies low enough to be energetically accessible. For states which become bound, ie cross the $\mathrm{CO}^{+} \mathrm{X}^{2} \Sigma^{+}$curve, within the range of bondlengths considered we have also attempted to determine the location of the corresponding bound states region of the curve using our bound state detecting program BOUND (Sarpal et al. 1991). This results, as can be seen from the figure, in curves that link the quasibound and bound states, but that these curves are often far from smooth. This lack of smoothness is not due to noise in our calculations but is caused by couplings to the (infinite) Rydberg series converging to the $\mathrm{CO}^{+} \mathrm{X}^{2} \Sigma^{+}$ionisation limit which couple with dissociative intruder states. Our curves therefore represent (approximate) diabats for these intruders.

Figures 1 and 2 show that the lowest resonance with ${ }^{1} \Pi,{ }^{1} \Delta,{ }^{3} \Sigma^{+}$and ${ }^{3} \Pi$ symmetries all cross the $\mathrm{CO}^{+}$ground state curve near $R=2.3 \mathrm{a}_{0}$. This means that any theoretical study of low-energy dissociative recombination of $\mathrm{CO}^{+}$will need to start by considering all these four curves even at the lowest energies.

As mentioned above, states with $\Sigma^{-}$spatial symmetry cannot couple to the target states of the $\Sigma^{+}$spatial symmetry. This means that the first series of resonance for both ${ }^{1} \Sigma^{-}$and ${ }^{3} \Sigma^{-}$symmetries actually have zero width and are therefore effectively bound states in the continuum. Table 3 gives energies and effective quantum numbers for these states. The data in table 3 has been organised to emphasise the near-degeneracy of singlet and triplet states. The small splitting between the singlets and triplets is to be expected for high Rydberg states where spin coupling of the outer electron to the core is weak. The two states, one singlet and one triplet, which are not given as pairs appear to be intruders which will therefore have much lower effective quantum numbers. 
Table 3. Energies, in Ryd, and effective quantum numbers for $\Sigma^{-}$bound states in the continuum at $R=2.132 \mathrm{a}_{0}$.

\begin{tabular}{cccc}
\hline \multicolumn{2}{c}{ Singlet states } & \multicolumn{2}{c}{ Triplet states } \\
Position & $\nu$ & Position & $\nu$ \\
0.1089 & 2.3631 & 0.1087 & 2.3620 \\
0.1849 & 3.1138 & 0.1845 & 3.1083 \\
0.2002 & 3.3757 & 0.2002 & 3.3753 \\
0.2256 & 4.0034 & 0.2256 & 4.0034 \\
0.2294 & 4.1306 & 0.2292 & 4.1249 \\
0.2359 & 4.3820 & 0.2358 & 4.3791 \\
& & 0.2463 & 4.8955 \\
0.2501 & 5.1373 & 0.2500 & 5.1331 \\
0.2535 & 5.3852 & 0.2536 & 5.3910 \\
0.2602 & 5.9999 & 0.2603 & 6.0054 \\
0.2615 & 6.1407 & 0.2614 & 6.1357 \\
0.2635 & 6.3870 & 0.2635 & 6.3898 \\
0.2684 & 7.1428 & 0.2684 & 7.1377 \\
0.2697 & 7.3881 & 0.2697 & 7.3904 \\
0.2729 & 8.1441 & 0.2729 & 8.1390 \\
0.2738 & 8.3888 & 0.2738 & 8.3909 \\
0.2744 & 8.5667 & & \\
0.2760 & 9.1450 & 0.2760 & 9.1398 \\
0.2766 & 9.3893 & 0.2767 & 9.3912 \\
\hline
\end{tabular}

\subsection{Electronic excitation}

Figures 3 and 4 present cross sections for electron impact electronic excitation of $\mathrm{CO}^{+}$. These cross sections were calculated by summing the contributions of singlet and triplet symmetries up to and including $\Delta$ using the frozen nuclei approximation and a $\mathrm{CO}^{+}$ bondlength of $2.132 \mathrm{a}_{0}$. Given that the nuclei in $\mathrm{CO}^{+}$move slowly compared to the electron impact the fixed nuclei approximation is probably reasonable starting point. Both figures show considerable structure. This structure is associated with the many resonances found in the system. These resonances are, at least at the lower energies considered, real but in practise their influence is washed out by the vibrational motion of the nuclei. This means that no particular significance should be given to the individual peaks and troughs shown in our cross section figures.

Figure 3 gives our predictions for excitation to the first excited state of $\mathrm{CO}^{+}$, the $\mathrm{A}^{2} \Pi$ state. As far as we know there is no previous data on this process for us to compare with. The one previous electron impact excitation measurement concerns electron impact dissociation.

Figure 4 presents our estimate of the electron impact dissociation cross section as a function of electron energy. It is well know that this process goes via electron impact 


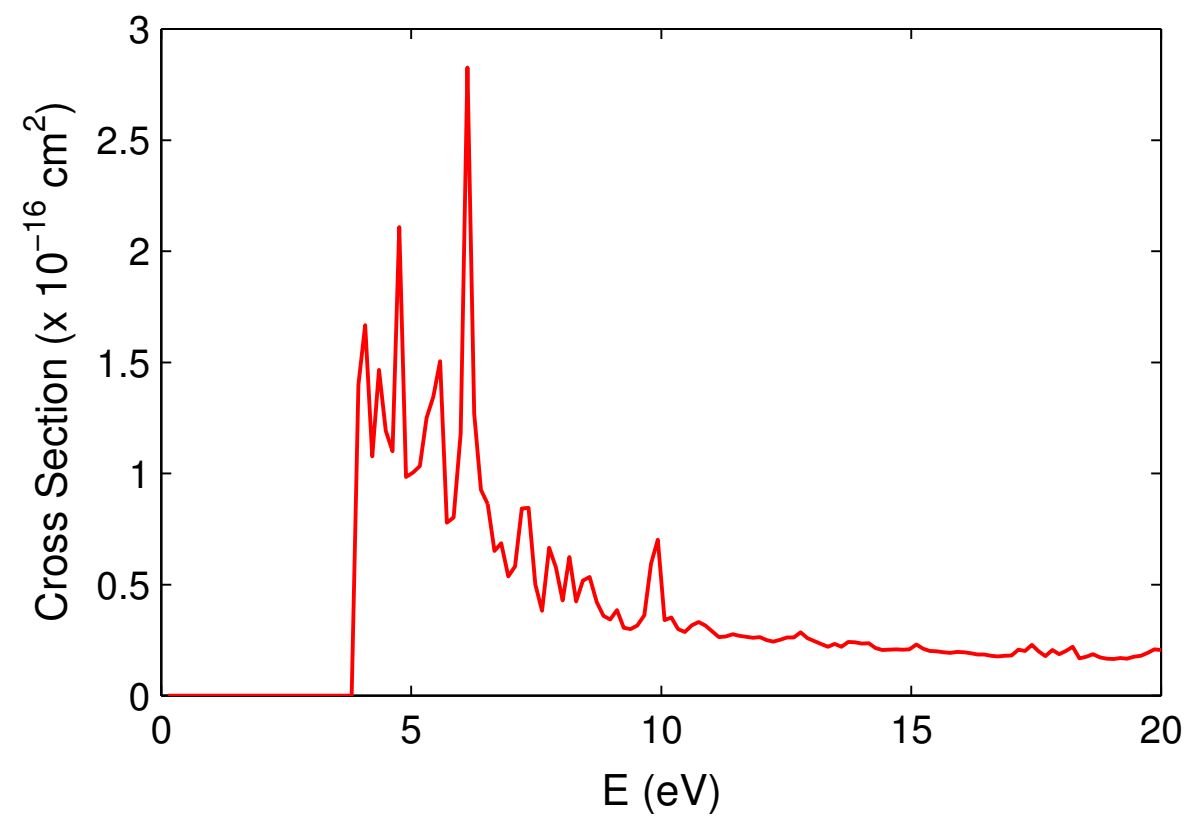

Figure 3. Electron impact excitation cross section for $\mathrm{CO}^{+} \mathrm{X}^{2} \Sigma^{+} \rightarrow \mathrm{A}^{2} \Pi$ calculated for a fixed bondlength of $R=2.132 \mathrm{a}_{0}$.

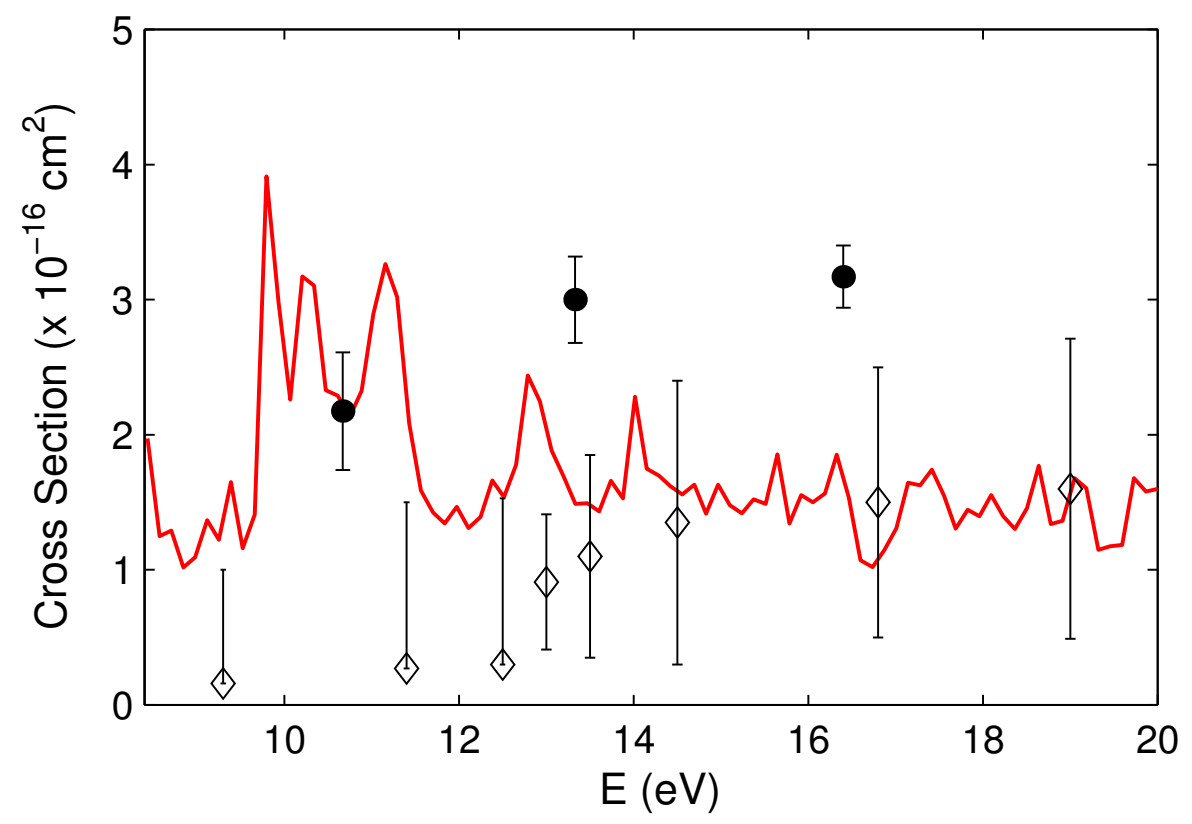

Figure 4. Estimated electron impact dissociation cross section for $\mathrm{CO}^{+}$calculated for a fixed bondlength of $R=2.132 \mathrm{a}_{0}$. Also shown are the measurements of Mitchell \& Hus (1985), filled circles, and Rosen et al. (1998), open diamonds. 
electronic excitation, particularly to dissociative electronic states. In estimating this cross section we have assumed that all electron impact electronic excitation which occurs above the dissociation threshold of $8.5 \mathrm{eV}$ leads to dissociation. This will clearly lead to an overestimate of the dissociation cross section, particularly close to the dissociation threshold. Conversely our calculations only include electronically excited states of $\mathrm{CO}^{+}$up to about $12.5 \mathrm{eV}$. At electron impact energies above this it is likely that our predictions will be an underestimate because of this. Despite these caveats our results are comparable to the experimental studies (Mitchell \& Hus 1985, Rosen et al. 1998) of this process. Given that our results will overestimate the amount of dissociation near the dissociation threshold, our results can be taken to be closer to those of Rosen et al. (1998) than Mitchell \& Hus (1985). To improve on our estimate would require both detailed consideration of nuclear motion, see Trevisan \& Tennyson (2001), and inclusion of many more excited states of $\mathrm{CO}^{+}$. Such a study is beyond the scope of this work.

\section{Conclusions}

We have used the UK R-matrix codes to consider electron collisions with the $\mathrm{CO}^{+}$ molecular ion. As is usual for electron collisions with positively charged ions, there are many series of Feshbach resonances associated with these collisions. The low-lying resonances identified are important for studies of dissociative recombination. Previous experience suggests that for a many electron target ion it is very hard to get the crossing point between such resonances and the ground state of the associated ion precisely (Schneider et al. 2000). However it has been shown (Tennyson 1996b, Rabadán \& Tennyson 1997, Schneider et al. 2000) that such error can be corrected for by shifts in the small, geometry independent, the quantum defect. An increase in the quantum defect in our calculation by about 0.05 is to be anticipated. Such an approach has been found to both give excellent results when compared with experiment (Schneider et al. 2000) and to be useful for predictions (Motapon et al. 2006).

We present data for electron impact electronic excitation of $\mathrm{CO}^{+}$. Here our results suggest that the measurements of the electron impact dissociation cross section by Rosen et al. (1998) is probably closer to the true result than that of Mitchell \& Hus (1985). Comparisons of electron impact electronic excitation cross sections between theory and experiment are difficult not only because of the theoretical approximations inherent in the calculations, here for example we have used the fixed nuclei approximation, but also because measured electronic excitation cross section often include cascade effects (Morgan \& Tennyson 1993) whereas calculations normally yield the direct excitation cross section only.

Finally we note that our calculations intrinsically contain information on a variety

of other processes involving continuum states of CO. It is our plan to use these wave functions to study electron impact rotational excitation of $\mathrm{CO}^{+}$as part of a study on the process for a variety of astronomical molecules. This can be done with confidence 
given the recent validation of our method (Faure et al. 2006).

\section{Acknowledgements}

We thank the Royal Society and the Indian National Science Academy for funding KC's visit to UCL during which this work was performed.

\section{References}

Burke P G \& Berrington K A, eds 1993 Atomic and Molecular Processes, an R-matrix Approach Institute of Physics Publishing Bristol.

Buttle P J A 1967 Phys. Rev. 160, 719-29.

Chakrabarti K \& Tennyson J 2006 J. Phys. B: At. Mol. Opt. Phys. 39, 1485-1497.

Erickson N R, Snell R L, Loren R B, Mundy L \& Plambeck R L 1981 Astrophys. J 245, L83-L86.

Faure A, Kokoouline V, Greene C H \& Tennyson J 2006 J. Phys. B: At. Mol. Opt. Phys. 39, 42614273.

Florescu-Mitchell A I \& Mitchel J B A 2006 Phys. Rep. 430, 277-374.

Fox J L \& Hac A 1999 J. Geophys. Res. 104, 24729-24737.

Fuente A \& Martin Pintado J 1997 Astrophys. J 477, L107.

Gorfinkiel J D, Morgan L A \& Tennyson J 2002 J. Phys. B: At. Mol. Opt. Phys. 35, 543-555.

Huber K P \& Herzberg G, eds 1979 Constants of Diatomic Molecules Van Nostrand Reinhold New York.

Kirby-Docken K \& Liu B 1977 J. Chem. Phys. 66, 4309-4316.

Lecointre J, Belic D S, Cherkani-Hassani H, Jureta J J \& Defrance P 2006 J. Phys. B: At. Mol. Opt. Phys. 39, 3275-3297.

McLaughlin B M, Ballance C P \& Berrington K A 2001 J. Phys. B: At. Mol. Opt. Phys. 34, L179L186.

McLean A D 1971 in "Conference on Potential Energy Surfaces in Chemistry", W.A. Lester Jr. (ed.), (IBM Research Laboratory, San Jose) p. 87.

Mitchell J B A \& Hus H 1985 J. Phys. B: At. Mol. Opt. Phys. 18, 547-555.

Morgan L A 1984 Comput. Phys. Commun. 31, 419.

Morgan L A \& Tennyson J 1993 J. Phys. B: At. Mol. Opt. Phys. 26, 2429-2441.

Motapon O, Fifirig M, Florescu A, Waffeu-Tamo F O, Crumeyrolle O, Varin-Breant G, Bultel A, Vervisch P, Tennyson J \& Schneider I F 2006 Plasma Phys. Science Technology 15, 23-32.

Noble C J \& Nesbet R K 1984 Comput. Phys. Commun. 33, 399.

Okada K \& Iwata S $2000 \mathrm{~J}$. Chem. Phys. 112, 1804.

Rabadán I \& Tennyson J 1996 J. Phys. B: At. Mol. Opt. Phys. 29, 3747-3761.

Rabadán I \& Tennyson J 1997 J. Phys. B: At. Mol. Opt. Phys. 30, 1975-1988. erratum 31, 4485-4487 (1998).

Rosen S, Peverall R, Larsson M, Le Padellec A, Semaniak J, Larson A, Stromholm C, van der Zande W J, Danared H \& Dunn G H 1998 Phys. Rev. A 57, 4462-4471.

Sarpal B K, Branchett S E, Tennyson J \& Morgan L A 1991 J. Phys. B: At. Mol. Opt. Phys. 24, 3685-3699.

Schneider I F, Rabadán I, Carata L, Tennyson J, Andersen L H \& Suzor-Weiner A 2000 J. Phys. B: At. Mol. Opt. Phys. 33, 4849-4861.

Storzer H, Stutzki J \& Sternberg A 1995 Astron. Astrophys. 296, L9-L12.

Tennyson J 1996 a J. Phys. B: At. Mol. Opt. Phys. 29, 1817-1828.

Tennyson J 1996b J. Phys. B: At. Mol. Opt. Phys. 29, 6185-6201.

Tennyson J, Burke P G \& Berrington K A 1987 Computer Phys. Comms. 47, 207-212.

Tennyson J \& Noble C J 1985 J. Phys. B: At. Mol. Phys. 18, 155-165. 
Trevisan C S \& Tennyson J 2001 J. Phys. B: At. Mol. Opt. Phys. 34, 2935-2949. 